\title{
Elegance as Complexity Reduction in Systems Design
}

\author{
Luca Iandoli $\mathbb{D},{ }^{1,2}$ Letizia Piantedosi, ${ }^{2}$ Alejandro Salado $\mathbb{D}^{3},{ }^{3}$ and Giuseppe Zollo ${ }^{2}$ \\ ${ }^{1}$ Division of Computer Science, Mathematics, and Science, St. John's University, New York City, NY, USA \\ ${ }^{2}$ Department of Industrial Engineering, University of Naples Federico II, Naples, Italy \\ ${ }^{3}$ Grado Department of Industrial and Systems Engineering, Virginia Tech, Blacksburg, VA, USA \\ Correspondence should be addressed to Luca Iandoli; liandoli@stevens.edu
}

Received 5 December 2017; Revised 5 May 2018; Accepted 13 May 2018; Published 26 August 2018

Academic Editor: Guido Caldarelli

Copyright $\odot 2018$ Luca Iandoli et al. This is an open access article distributed under the Creative Commons Attribution License, which permits unrestricted use, distribution, and reproduction in any medium, provided the original work is properly cited.

\begin{abstract}
Elegance is often invoked as a characteristic of good design, but it cannot be pursued as a design objective because of the absence of actionable definitions that can be translated into design strategies and metrics. In this work, we analyze elegance in the context of systems engineering using a perspective that integrates visual art, Gestalt psychology, neuroscience, and complexity theory. In particular, we measure elegance as effective complexity and theorize that it can be achieved by a process of complexity resolution based on the adoption of eight visual heuristics. We present an empirical study in which a sample of systems engineers were asked to assess alternative representations of a same system and show that effective complexity is strongly correlated to perceived elegance and systems effectiveness. Our results are consistent with independent findings obtained in other fields including design and psychology of perception showing that good design must embed an effective level of complexity achievable through a mix of familiarity and novelty.
\end{abstract}

\section{Introduction}

Research in neuroscience has made substantial progress in the last decade in improving our understanding about how our brain produces knowledge by extracting meaningful patterns from the flow of data that hit our senses. The complexity of the brain has evolved in time integrating the older subconscious and emotional strata with the more recent conscious and rational layers [1]. As a result, the human brain's ability to extract meaning from the sensory world is not only built on the analytical skills of the higher-level layers but also on fast-thinking heuristics [2] based on a priori psychological constructs [3] and activated by gut feelings developed through environmental adaptation [4]. Such a mix of emotional and analytical thinking is particularly evident in the way the human brain perceives art.

Drawing from Gestalt psychology [3] and through the use of advanced MRI techniques to detect cerebral activity, several neuroscientists $[1,5,6]$ claim that the aesthetic enjoyment we experience in front of a masterpiece is an exceptional empirical situation to study a key human ability, that is, how we elicit meaningful patterns to reduce the complexity determined by ambiguous information. According to these studies, understanding a painting is the result of the application of specific cognitive strategies and of a system of biochemical incentives that reward our pattern recognition ability [7].

Building on this perspective and findings, we argue that if evolution has hardwired the human brain with cognitive abilities for the appreciation of beauty, the aesthetic value of a representation must also matter for other practical purposes. In the engineering realm, systems engineering has been found valuable in dealing with complex system because of its inherent capability to reduce complexity [8]. In particular, system architecting, which enables structuring a problem into manageable pieces, is instrumental in identifying and using patterns that yield good, simple, elegant architectures [9-11]. Thus, both aesthetic experience and engineering systems architecture seem to have in common the objective of reducing complexity and recent work related to the application of systems engineering practices to artistic composition shows that these two perspectives can be successfully integrated [12]. 
TABLE 1: Artistic strategies for reducing complexity.

\begin{tabular}{l} 
Noise-killing strategies \\
\hline (X1) Subtract details: eliminate unnecessary and uninformative \\
details (e.g., by elementary geometric shapes, minimalist \\
representation, sketched figures, and abstraction)
\end{tabular}

(X2) Symmetry: can be vertical, horizontal, applied to the entire image, or to part of it

(X3) Grouping: use of clusters to group visual elements and arrange them in the image to communicate a sense of order or relationship (by similarity, spatial proximity, juxtaposing different groups, etc.)

(X4) Split: use of informational hierarchy in the image in which visual elements are present at different levels (e.g., recursivity, as in the use of fractal forms, and background/foreground)

In order to explore this idea further, based on findings and theories developed in design [13-15], Gestalt psychology $[3,16]$, and neuroaesthetics $[1,5,6]$, we identified eight visual strategies that correspond to basic heuristics through which drawings and paintings can be created and interpreted (seven of them are listed in Table 1). These strategies can be organized into two groups: noise-killing strategies, aimed at simplifying reality by suppressing supposedly irrelevant stimuli (noise), and add-meaning strategies, whose objective is to add information when we realize our interpretation is too simple to explain reality or deliver the expected performance. We theorize that complexity reduction is achieved through the application by trial and error of both types of strategies until an optimal level of complexity is achieved (effective complexity [17]).

Gell-Mann and Lloyd [17] define effective complexity as the shortest description of the perceived regularities in a system. A completely random sequence of data has effective complexity equal to zero. The same would happen for a completely ordered sequence, such as a string containing only one symbol repeated many times. Sequences exhibiting some regularities would be in an intermediate region between total order and complete disorder, so their effective complexity is higher than zero. In this work, we will use effective complexity as a metric to assess the application of the seven visual heuristic strategies: specifically, we will show that noisekilling strategies are aimed at increasing effective complexity through simplification, that is, by looking for familiar input in the available stimuli; similarly, add-meaning strategies are aimed at increasing effective complexity through complexification, that is, through the discovery of new order in unfamiliar situations.

Interestingly, we found out that there is an overlap between the competences that artists and system architects need to master to design artifacts whose internal complexity matches the external complexity of the task they are designed to address [18-20].

In this paper, we test this hypothesis further. In particular, we present an empirical study in which we surveyed a
Add-meaning strategies

(X5) Emphasize: assigning importance to elements by deliberate distortion (e.g., exaggerated proportions and emphasis on characterizing traits, as in caricature)

(X6) Power of center: creation of a strong visual center toward which the author intends to draw the observer's attention. The center is clearly identifiable by graphic elements directing attention such as direction lines, strong colors, and shapes or other graphical effects that radiates from the center

(X7) Contrast and balance: creation of tension between elements that are supposed to be in relationship by contrast of color or composition (e.g., use of contrast tones, use complementary colors, and juxtaposition of shapes and elements across some axis to balance for visual weight)

sample of systems engineers about the perceived effectiveness and elegance of alternative architectures of a same system. Our results show that systems architecture elegance can be measured as effective complexity [17]. We also found out that the experts in our sample perceived the most elegant architectures as the most effective and that elegance is achieved when all the visual strategies reported in Table 1 are applied in the design of the system representation.

The remainder of the paper is organized as follows. Section 2 describes the methodology. Section 3 presents the results of the survey. A discussion of the results is provided in Section 4. Conclusions are provided in Section 4.

\section{Methodology}

2.1. Design. This research was designed to test the following hypotheses:

(1) Perceived implementation effectiveness is correlated to the structural complexity of the architecture.

(2) Perceived elegance is correlated with the effective complexity of the architecture.

(3) Effective complexity is higher when both noisekilling and add-meaning strategies are used more intensively.

A sample of 54 systems engineering experts was presented with various candidate architectures of an automated teller machine (ATM); specifically, functional architectures have been used. Subjects were asked to rank the architectures based either on their perceived implementation effectiveness or on their perceived elegance (no definition of elegance was however given to the participants). Each participant was randomly presented with only one of the evaluations in order to avoid temporal precedence biases. Eight architectures were developed using different strength levels of the strategies reported in Table 1. All the architectures followed the same 
drawing pattern in order to isolate the effect of the artistic strategies.

Response data, together with demographic information, were collected via an online survey over a period of two weeks; each participant completed his or her task within 15 minutes in a single individual session. Participants worked autonomously, and there was no interaction between the participants and the researchers.

The ATM was selected to guarantee that the respondents were familiar with the operation and objectives of the system of interest. Besides, our subjects were experienced systems engineers that were familiar with systems engineering representation and notation; finally, the ATM system description we used in the empirical materials describes a traditional ATM service. These measures help in reducing the risk of biased evaluations that are obtained in single-shot assessments (assessments with just one evaluation phase without prior familiarization, see Section 2.3) and that are likely to surface when the evaluation concerns systems characterized by high novelty or innovativeness [21, 22].

2.2. Factors. The independent variable in this investigation is the set of artistic strategies that were used to develop the various ATM architectures. The strength or presence of the eight strategies in each architecture was assessed by an expert on a seven-point scale (1 corresponded to the "lowest level" and 7 corresponded to the "highest level"). Each level of the scale was anchored to descriptors determined through the construction of an evaluation rubric (see Supplementary 1).

Two dependent variables were measured: perceived implementation effectiveness and perceived elegance. Participants were required to rank the alternative designs in terms of both variables using a 1 to 8 ordinal scale, where 1 indicates the most preferred and 8 indicates the least preferred.

In the survey, we did not provide any specific objective or requirement for the architectures because the goal was to measure structural perception as opposed to a more rational/technical analysis of the system. To compensate in part for this constraint, we considered a system of interest (i.e., the ATM) whose overall purpose and user's needs are known by most adults. The time to complete the survey was limited to 15 minutes, much less than what would be necessary in a real-life assessment of a system's architecture. Again, this limitation was enforced to capture perception and to prevent subjects from engaging in a more in-depth technical analysis.

2.3. Materials. The survey was divided into two parts (Supplementary 2). The first part contained a demographic survey to collect information about the professional experience and educational background of the participants. The second part consisted of the eight ATM system architectures and two rank ordering questions, one addressing perceived implementation effectiveness and one addressing perceived elegance (note: each participant was only given one of the two questions randomly). Two examples of diagrams representing the ATM system architecture are depicted in Figure 1.

The diagrams were represented as box and arrows workflow and did not contain formal notations because the use of a more formal description could have affected the ranking depending on the level of expertise or familiarity of the participants with the specific modeling language. Every diagram was identified through a letter from $\mathrm{A}$ to $\mathrm{H}$.

The diagrams were attached to the survey in a downloadable MS PowerPoint file and randomly displayed in a single slide as in Figure 2. Participants could click on each miniature to enlarge the image and inspect the architecture (Figure 1).

The survey consisted of a single question asking participants to rank the eight diagrams in terms of either perceived implementation effectiveness or perceived elegance. Both surveys were administered online via Qualtrics and could be accessed via a web link provided to the subjects.

The architectures were designed by one of the authors of this paper to deliberately incorporate the strategies $X_{\mathrm{i}}$ at different levels of intensity, while another author carried out the assessment reported in Table 2 independently, using the assessment tool reported in Supplementary 1. The strength levels of the strategies corresponding to the architectures depicted in Figure 2 are reported in Table 2.

2.4. Subjects. Fifty-four complete and usable surveys were collected. Participants did not receive any compensation. Participation was anonymous. Only the answers provided by participants belonging to one or more of the following categories have been eventually included in the analysis:

(i) Self-identified systems engineers with at least 5 years of professional experience: a practicing systems engineer is defined as an individual who executes some or all of the activities described in [8] as part of his/her professional work

(ii) Systems engineering faculty

(iii) Doctoral students in systems engineering

2.5. Effective Complexity Metric. No definition for elegance was provided to the participants. A simple and shared definition of elegance is notoriously difficult to find, so we relied on the subjective interpretation that participants had of the concept, typically interpreted in terms of a mix of ingenuity and simplicity. Implementation effectiveness was defined as the quality of each architecture to be conducive to easy implementation.

Structural complexity was computed using two different metrics: cyclomatic complexity [23] and topological complexity [24].

The cyclomatic complexity of a graph $G$ with $m$ edges and $n$ nodes is given by

$$
V(G)=m-n+1
$$




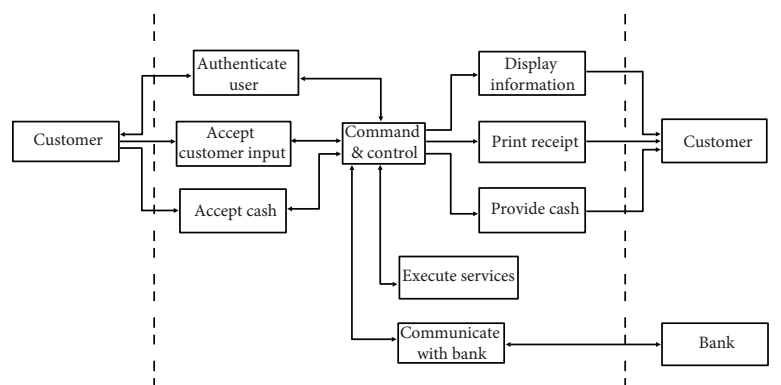

(g)

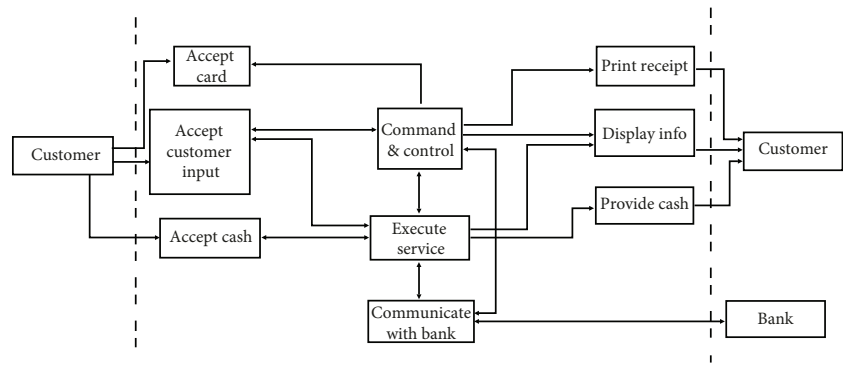

(h)

FIgURE 1: Examples of two architectures.

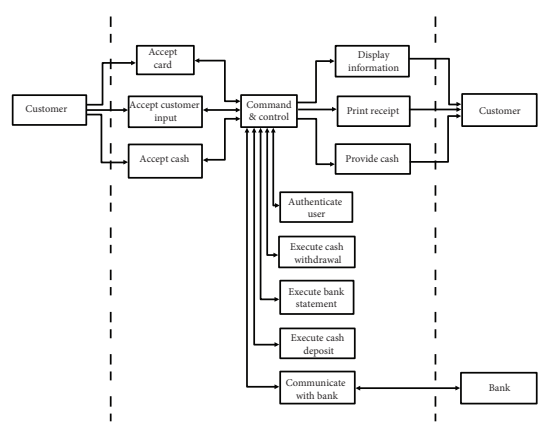

(a)

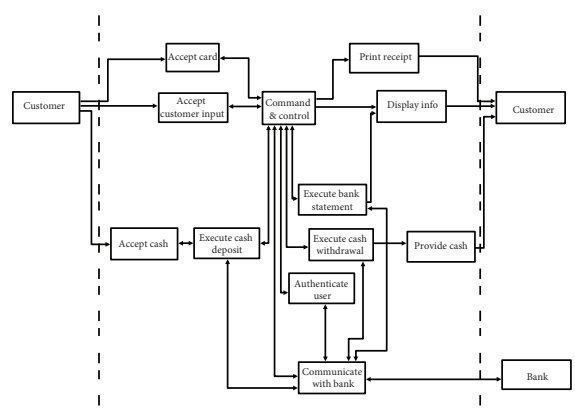

(d)

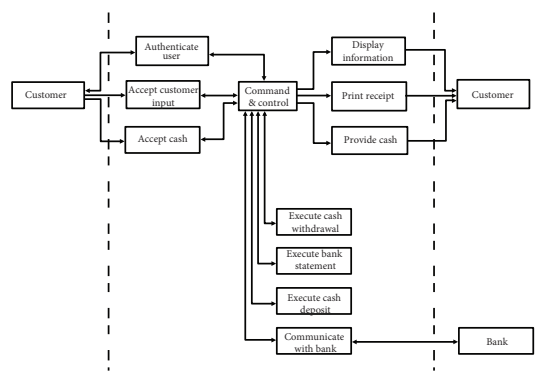

(b)

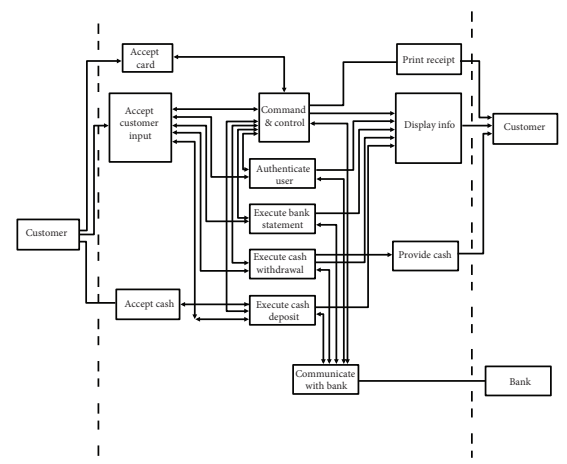

(e)

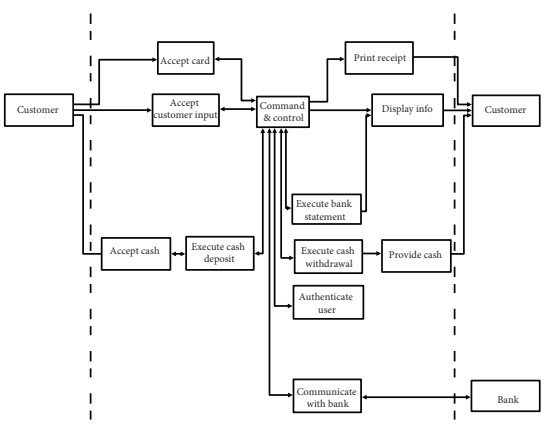

(c)

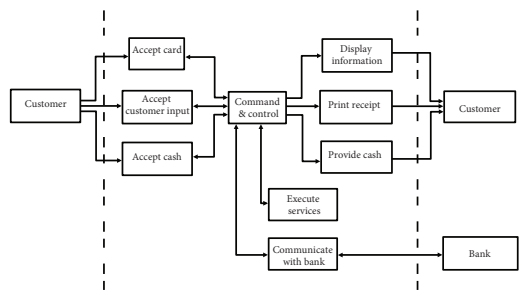

(f)

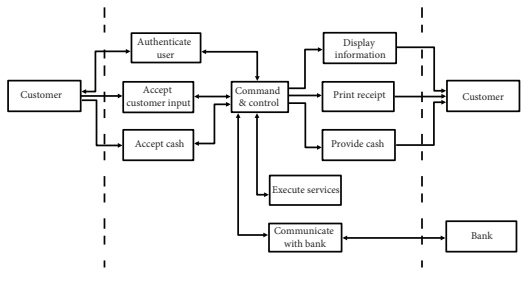

(g)

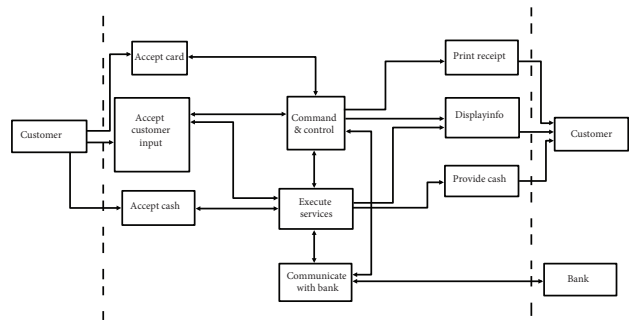

(h)

FIgURE 2: Alternative systems representations of an ATM used in the survey.

This metric counts the number of linearly independent cycles present in the network. Fewer cycles within the network should result in easier implementability.

Topological complexity is expressed as

$$
C_{3}=\frac{E(A)}{n},
$$

where $E(A)$ represents the graph energy of a network, defined as the sum of singular values $\sigma_{i}$ of the adjacency matrix:

$$
E(A)=\sum_{i=1}^{n} \sigma_{i} .
$$

Graph energy reveals the complexity of the structural dependency among the components. Topological 
TABLE 2: Strength levels of the artistic strategies in the eight ATM architectures.

\begin{tabular}{lccccccc}
\hline \multirow{2}{*}{ Diagram } & Subtract details $X 1$ & Symmetry $X 2$ & Grouping $X 3$ & Split $X 4$ & Emphasize $X 5$ & Power of center X6 & Contrast/balance $X 7$ \\
\hline A & 6 & 7 & 5 & 4 & 4 & 7 & 7 \\
B & 6 & 6 & 5 & 4 & 7 & 7 & 7 \\
C & 4 & 3 & 2 & 2 & 2 & 4 & 4 \\
D & 3 & 2 & 2 & 2 & 2 & 3 & 3 \\
E & 2 & 6 & 1 & 1 & 1 & 1 & 7 \\
F & 6.67 & 7 & 6 & 6 & 5 & 7 & 7 \\
G & 6.67 & 7 & 6 & 6 & 7 & 7 & 1 \\
H & 2.67 & 6 & 4 & 3 & 1 & 1 & 7 \\
\hline
\end{tabular}
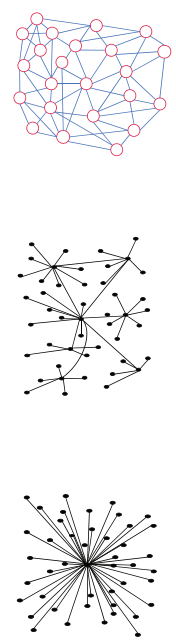

“Distributed" architecture

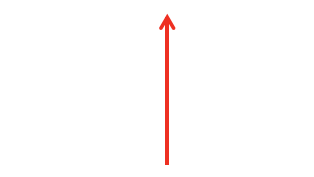

"Hierarchical" architecture

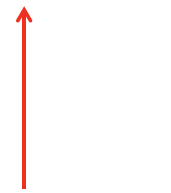

Centralized architecture
Increasing topological complexity

$\left(C_{3}\right)$

Figure 3: Architectural patterns defined in terms of topological complexity (source: Sinha [24]).

complexity metric $C_{3}$ values are associated to these system architectural patterns:

$$
\begin{gathered}
C_{3}<1 \longrightarrow \text { centralized architecture, } \\
1 \leq C_{3}<2 \longrightarrow \text { hierarchical architecture, } \\
C_{3} \geq 2 \longrightarrow \text { distributed architecture. }
\end{gathered}
$$

A conceptual example of how topological complexity relates to architectural patterns is shown in Figure 3.

\section{Results}

The questionnaire was submitted to experts belonging to online groups of systems engineering and by individual email invitation. Eventually, we collected 54 usable surveys. The average ranking of elegance and perceived implementation effectiveness is reported in Table 3. The sample for each question is 27 subjects because participants were randomly assigned to answer either the elegance or the implementation effectiveness question.

The two rankings are perfectly correlated (Spearman rank correlation is equal to 1 ), which means that independent
TABLE 3: Average ranking of perceived implementation effectiveness and perceived elegance $(N=27)$ on a 7 -point Likert scale with $1=$ most preferred and $7=$ least preferred.

\begin{tabular}{lcc}
\hline $\begin{array}{l}\text { System } \\
\text { diagram }\end{array}$ & $\begin{array}{c}\text { Perceived implementation } \\
\text { effectiveness }(n=27)\end{array}$ & $\begin{array}{c}\text { Perceived elegance } \\
(n=27)\end{array}$ \\
\hline A & 4.2 & 3.9 \\
B & 3.8 & 3.5 \\
C & 5.5 & 5.2 \\
D & 5.9 & 6.4 \\
E & 6.5 & 7.1 \\
F & 3.1 & 3.0 \\
G & 2.6 & 2.7 \\
H & 4.3 & 4.2 \\
\hline
\end{tabular}

experts considered the most elegant diagrams as the ones that turned out to be also easier to implement.

The first experimental hypothesis predicts the presence of correlation between preference judgments on implementation effectiveness and structural complexity measures (most preferred diagrams are the least structurally complex). 
TABLE 4: Data for Spearman correlation coefficient calculation.

\begin{tabular}{lcccc}
\hline Diagram & $\begin{array}{c}\text { Cyclomatic } \\
\text { complexity } \\
(\text { CC) }\end{array}$ & $\begin{array}{c}\text { Perceive } \\
\text { implementation } \\
\text { effectiveness }(P)\end{array}$ & $\begin{array}{c}\text { Rank } \\
\text { CC }\end{array}$ & $\begin{array}{c}\text { Rank } \\
P\end{array}$ \\
\hline A & 14 & 4.2 & 3.5 & 4 \\
B & 14 & 3.8 & 3.5 & 3 \\
C & 15 & 5.5 & 5.5 & 6 \\
D & 23 & 5.9 & 7 & 7 \\
E & 34 & 6.5 & 8 & 8 \\
F & 11 & 3.1 & 1 & 2 \\
G & 12 & 2.6 & 2 & 1 \\
H & 15 & 4.3 & 5.5 & 5 \\
\hline
\end{tabular}

TABLe 5: Data for Spearman correlation coefficient calculation.

\begin{tabular}{lcccc}
\hline Diagram & $\begin{array}{c}\text { Topological } \\
\text { complexity }\left(C_{3}\right)\end{array}$ & $\begin{array}{c}\text { Perceived } \\
\text { implementation } \\
\text { effectiveness }(P)\end{array}$ & Rank $C_{3}$ & $\begin{array}{c}\text { Rank } \\
P\end{array}$ \\
\hline A & 0.74 & 4.2 & 1 & 4 \\
B & 0.86 & 3.8 & 2 & 3 \\
C & 0.998 & 5.5 & 5 & 6 \\
D & 1.14 & 5.9 & 6 & 7 \\
E & 1.26 & 6.5 & 8 & 8 \\
F & 0.92 & 3.1 & 3.5 & 2 \\
G & 0.92 & 2.6 & 3.5 & 1 \\
H & 1.15 & 4.3 & 7 & 5 \\
\hline
\end{tabular}

First, Spearman rank correlation coefficient was calculated between the average preference rankings and cyclomatic complexity values (Table 4). Computing Spearman correlation coefficient using the data contained in Table 4, we obtain

$$
\rho_{s}=1-\left(\frac{6 \sum_{i=1}^{n} D_{i}^{2}}{n\left(n^{2}-1\right)}\right)=1-\left(\frac{6 * 3}{8(64-1)}\right)=0.96
$$

where $D_{\mathrm{i}}=$ rank $\mathrm{CC}_{\mathrm{i}}-\operatorname{rank} P_{\mathrm{i}}$ and $n=8$. The correlation is significant at $p=0.05$, so hypothesis 1 is confirmed when structural complexity is measured as cyclomatic complexity.

We retested the hypothesis using topological complexity. Table 5 reports the values of perceived effectiveness implementation and topological complexity for each diagram and the associated rankings. Spearman correlation in this case is equal to 0.71 , still significant at $p=0.05$ for $n=8$. Thus, hypothesis 1 is verified also when using topological complexity.

Hypothesis 2 states that perceived elegance is correlated with effective complexity. Effective complexity for each diagram was calculated using the $L M C$ measure proposed by Piqueira and de Mattos [25]. This metric is inspired to Gell-Man and Lloyd [17] definition of effective complexity (EC) of an entity as the length of a highly compressed description of its regularities. LMC measure is defined as
TABLE 6: Entropy calculation for each diagram.

\begin{tabular}{lccccc}
\hline Diagram & $n$ & $m$ & $M$ & $p$ & $S$ \\
\hline A & 14 & 27 & 182 & 0.148 & 110.237 \\
B & 13 & 26 & 156 & 0.1667 & 101.403 \\
C & 14 & 29 & 182 & 0.159 & 115.157 \\
D & 14 & 36 & 182 & 0.198 & 130.587 \\
E & 14 & 47 & 182 & 0.258 & 149.983 \\
F & 11 & 21 & 110 & 0.191 & 77.371 \\
G & 11 & 22 & 110 & 0.200 & 79.412 \\
H & 11 & 26 & 110 & 0.236 & 86.783 \\
\hline
\end{tabular}

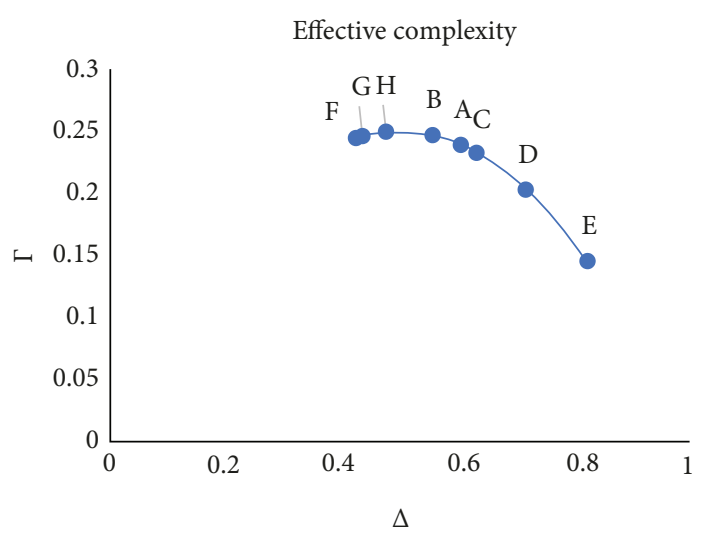

FIgURe 4: Effective complexity curve.

a weighted geometric average combination of order and disorder measures.

$$
\Gamma_{1 k}=(1-\Delta)(\Delta)^{k}
$$

where $\Delta$ is a measure of disorder defined as relative entropy as follows:

$$
\Delta=\frac{S}{S_{\max }},
$$

where $S$ is the entropy of the system. We characterized $S$ as an entropy measure based on the topological configuration of a network [26].

$$
S(N, p)=-M\left[p \log _{2} p+(1-p) \log _{2}(1-p)\right],
$$

where $M$ is the maximum number of possible edges in the network, which for an oriented graph with $n$ nodes is equal to $n(n-1)$. Link probability $p$ is equal to the ratio between the number of actual connections $m$ tall the potential $M$ connections.

Table 6 shows these measurements for each of the eight diagrams together with the corresponding entropy values.

$S_{\max }$ is the maximum value of entropy which is equal to $M$ when $p=0.5$. Since the experts were considering relative ranking based on the available alternatives, we used the highest value of $M$ in our sample $(M=182$, see Table 6).

Using these data, we constructed the effective complexity curve (Figure 4, from the data in Table 7) 
TABLE 7: Effective complexity calculation and ranking for each diagram.

\begin{tabular}{lcc}
\hline Chart & Disorder $\left(S / S_{\max }\right)$ & Effective complexity $(L M C)$ \\
\hline F & 0.425 & 0.244 \\
G & 0.436 & 0.246 \\
H & 0.477 & 0.249 \\
B & 0.557 & 0.245 \\
A & 0.606 & 0.239 \\
C & 0.633 & 0.232 \\
D & 0.717 & 0.203 \\
E & 0.824 & 0.145 \\
\hline
\end{tabular}

as a function of $\Delta$ and ranked the diagrams in terms of their effective complexity. The curve reproduces the inverse U-shaped curve theorized by Gell-Man and Lloyd [17] according to which the highest level of effective complexity is the result of a mix of predictable order and understandable disorder.

The value of Spearman's correlation between EC and elegance ranking (Table 8 ) is equal to 0.74 , which is significant at $p=0.05$ for $n=8$. Hypothesis 2 is then supported.

Hypothesis 3 states that higher level of effective complexity is achieved when both noise-killing and add-meaning strategies are used at higher level of intensity.

The diagrams that are perceived as the most elegant, such as G, F, A, and B, are the ones that in Table 2 score high both on the strategies and on the effective complexity metric, with $\mathrm{H}$ being the only exception (lower score on strategies, but high score on EC). This correlation can be offered as evidence supporting hypothesis 3 .

Spearman correlation coefficient was calculated between the vector norms of the strategy assessments corresponding to the architectures (Table 2) and elegance ranking (Table 9). The correlation is equal to 0.98 , and it is significant at $p=0.05$ for $n=8$. Thus, the most elegant diagrams incorporate the highest intensity levels of the visual strategies.

However, in order to discount for potential correlation between the original strategy variables, we performed a principal component analysis. The loading factors over two principal components are reported in Table 10. The first two components $Y 1$ and $Y 2$ explain $94.16 \%$ of the variance in the sample where

$$
\begin{aligned}
Y 1= & 0.41(X 1)+0.2643(X 2)+0.3893(X 3)+0.3907(X 4) \\
& +0.3854(X 5)+0.3932(X 6)+0.3932(X 7), \\
Y 2= & 0.1486(X 1)-0.8076(X 2)-0.2516(X 3)-0.1722(X 4) \\
& +0.1766(X 5)+0.3175(X 6)+0.3175(X 7) .
\end{aligned}
$$

Figure 5 reports the 8 diagrams on the bidimensional space determined by the principal components. It is possible to observe that the most elegant system diagrams, such as A, F, G, and B, are on the right side. They are very close to the horizontal axis, which corresponds to variable $Y 1$, on which
TABLE 8: Data for Spearman correlation coefficient calculation.

\begin{tabular}{lcccc}
\hline Diagram & Elegance & $\begin{array}{c}\text { Effective } \\
\text { complexity }\end{array}$ & $\begin{array}{c}\text { Elegance } \\
\text { ranking }\end{array}$ & $\begin{array}{c}\text { Effective } \\
\text { complexity } \\
\text { ranking }\end{array}$ \\
\hline A & 3.9 & 0.239 & 4 & 5 \\
B & 3.5 & 0.245 & 3 & 3 \\
C & 5.2 & 0.232 & 6 & 6 \\
D & 6.4 & 0.203 & 7 & 7 \\
E & 7.1 & 0.145 & 8 & 8 \\
F & 3.0 & 0.244 & 2 & 4 \\
G & 2.7 & 0.246 & 1 & 2 \\
H & 4.2 & 0.249 & 5 & 1 \\
\hline & & & &
\end{tabular}

TABLE 9: Data for Spearman correlation coefficient calculation.

\begin{tabular}{lcccc}
\hline Diagram & $\begin{array}{c}\text { Vector } \\
\text { norms }\end{array}$ & Elegance & $\begin{array}{c}\text { Vector norms } \\
\text { ranking }\end{array}$ & $\begin{array}{c}\text { Elegance } \\
\text { ranking }\end{array}$ \\
\hline A & 15.5 & 3.9 & 5 & 4 \\
B & 16.1 & 3.5 & 6 & 3 \\
C & 8.3 & 5.2 & 3 & 6 \\
D & 6.6 & 6.4 & 1 & 7 \\
E & 6.7 & 7.1 & 2 & 8 \\
F & 17.0 & 3.0 & 7 & 2 \\
G & 17.7 & 2.7 & 8 & 1 \\
H & 8.4 & 4.2 & 4 & 5 \\
\hline
\end{tabular}

all the seven strategies are projected with more or less the same load factor. The most inelegant architecture, such as $\mathrm{C}, \mathrm{D}$, and $\mathrm{E}$, lies on the opposite side of the plane.

\section{Discussion and Conclusions}

The results presented in this paper suggest that elegance can be described in terms of effective complexity.

First, our results show a high degree of correlation between elegance and structural complexity, as well as between elegance and implementation effectiveness. In other words, the most elegant design is perceived both as structurally simple and as conducive to more effective and easy implementation.

Second, the correlation between perceived elegance and effective complexity shows that the level of elegance can be understood in terms of "desirable" complexity. While generally elegance is considered as a very subjective and vague concept, our results show that the effective complexity metric used in this study was highly predictive of the elegance ranking provided by the experts. The proposed metric assesses complexity as a balanced mix of familiar and discoverable order, with order measured in terms of entropy as defined in information theory.

Based on this result, we speculate that understanding and resolving complexity is a dynamic process in which individuals adopt noise-killing heuristics to simplify the problem 
TABLe 10: Principal components analysis.

\begin{tabular}{lcccccccr}
\hline Diagrams & & & & & & & & \\
& $\mathrm{A}$ & $\mathrm{B}$ & $\mathrm{C}$ & $\mathrm{D}$ & $\mathrm{E}$ & $\mathrm{F}$ & $\mathrm{G}$ & -1.8805 \\
\hline Y1 & 1.5715 & 1.8962 & -1.5962 & -2.2354 & -3.0415 & 2489 & 2.7969 & -1.3609 \\
$Y 2$ & -0.1329 & 0.4977 & 1119 & 1.2275 & -0.8415 & -0.325 & -0.184 & - \\
\hline
\end{tabular}

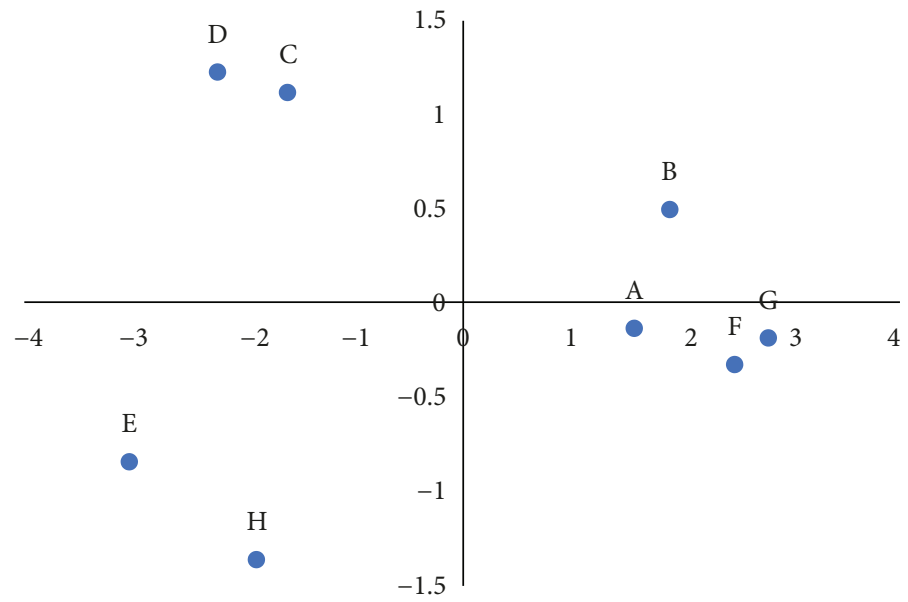

Figure 5: Principal component graph.

representation. On the other hand, when the representation becomes too simple and potentially unable to deliver the expected performance, we resort to add-meaning heuristics that eventually make the representation more complex, but also more meaningful.

This process may proceed by trial and error through the adoption of the seven artistic strategies until a sweet spot is achieved in terms of ideal mix of order and disorder. Of course, additional empirical work is needed to test this hypothesis by focusing on how subjects actually design systems and not only on how experts assess already made system architectures, as it happens in this work.

Elegance in this perspective can be considered as complexity reduction, as a tension toward simplicity that is balanced by an opposite tension toward the achievement of some level of meaningful complexity.

The same idea has been surprisingly mentioned in different fields. In design, Norman stated that good design always needs some complexity [14] and Maeda affirmed that good design is a mix of novelty and familiarity [13]. Following the MAYA design principle ("Most Advanced Yet Acceptable"), Hekkert et al. [15] showed empirically that familiarity ("typicality") and novelty are jointly effective in explaining the aesthetic preference in consumer products but also that they cancel each other out. Namely, they found in three studies that subjects "prefer novel designs as long as the novelty does not affect typicality, or, phrased differently, they prefer typicality given that this is not to the detriment of novelty" (p. 111). These results have been replicated in other design studies as well [27].

These works refer to previous research in psychology of perception. Berlyne [28] found that "The hedonic value of complex stimuli tends to rise as they become less novel while the opposite holds for simple stimuli" (p. 284). Berlyne speculated that the familiarity-novelty tradeoff is driven by two antagonist factors: a tedium factor that tends to be predominant when a simple stimulus is encountered repeatedly and a positive-habituation factor that instead prevails during repetition of complex stimulus.

Suggestively, semiologists and art critics of the caliber of Barthes extended this observation to art by arguing that art is a system without noise and that in great work of art, no unit of meaning gets wasted [29], implying again that artists tend toward efficient use of information in maximizing meaningfulness. While it takes a significant leap to extend these findings from the visual stimuli provided in lab settings to the fruition of complex artistic products in the real world, studies in neuroscience using fMRI [30] show that different types of representational paintings, such as landscapes and portraits, activated specific brain areas, whereas exposure to abstract art causes the activation of multiple brain areas at the same time. These observations provide some evidence that familiarity and novelty can trigger different neural and cognitive reactions (recognition of known forms VS exploration of novel forms). We then speculate that when simple familiar stimuli are mixed with more puzzling ones, as it may happen in innovative artistic representations, our brain works to integrate both types of information and that successful integration is eventually associated to higher hedonic value. The validation of this conjecture and its extension to design and art require additional research, and we suggest that this convergence of results between art, design, and psychology of perception offers a promising perspective for future investigation. 
For instance, we expect that similar results could be obtained by replicating this study with different types of visual stimuli and subjects. In particular, the methodology proposed in this paper could be applied to the analysis and design of user interface, such as in control panels, information dashboard, data visualization devices, and app or web site design.

The selection of the ATM and the use of functional architectures yield two potential limitations, in addition to the obvious - the generalizability issues that we already discussed in Section 2. First, we have assumed that expert perception of implementation effectiveness and elegance is independent of the actual system but is primarily affected by the underlying architectural patterns. This assumption is not arbitrary, though. On the contrary, it is grounded on the principles of system architecture [31], on collective experiences using architecture heuristics [9], and on the concept of architectural patterns [32]. However, our study does not explicitly explore the effect of this factor. Second, a system architecture has several dimensions, one of which is the functional one [31]. We have assumed that expert perception of implementation effectiveness and elegance is independent of the architectural dimension. We have grounded this assumption on the fact that the different dimensions share the same underlying relational structure $[31,33]$. However, our study does not explicitly explore the effect of this factor. In addition, the coupling between various architectural dimensions [9] is also not explored. These limitations will be central to future work.

\section{Conflicts of Interest}

All authors declare that they do not bear any conflict of interest regarding the publication of this paper and that their professional judgment concerning the validity of research is not influenced by secondary interests including financial gain or other forms of personal advantage or constraint.

\section{Supplementary Materials}

Supplementary 1. Strategies evaluation.

Supplementary 2. Elegance system architecture.

Supplementary 3. Alternative systems representations of an ATM used in the survey.

\section{References}

[1] V. S. Ramachandran, The Tell-Tale Brain: A Neuroscientist's Quest for What Makes Us Human, W. W. Norton \& Company, New York, NY, 2011.

[2] D. Kahneman, Thinking, Fast and Slow, Farrar Straus \& Giroux, New York, 2011.

[3] K. Koffka, Principles of Gestalt Psychology, vol. 44, Routledge \& Kegan Paul, London, 1935.

[4] G. Gigerenzer and G. Feelings, The Intelligence of the Unconscious, Penguin Group USA, London, 2007.

[5] E. R. Kandel, Reductionism in art and brain science: bridging the two cultures, Columbia University Press, New York, 2016.
[6] S. Zeki, Splendors and Miseries of the Brain: Love, Creativity, and the Quest for Human Happiness, John Wiley \& Sons, Ltd., West Sussex, UK, 2008.

[7] A. A. A. Salah and A. A. Salah, "Technoscience art: a bridge between neuroesthetics and art history?," Review of General Psychology, vol. 12, no. 2, pp. 147-158, 2008.

[8] INCOSE, Systems Engineering Handbook: A Guide for System Life Cycle Processes and Activities, version 4.0 ed, John Wiley and Sons, Inc., Hoboken, NJ, USA, 2015.

[9] M. W. Maier and E. Rechtin, The Art of System Architecting, CRC Press, 2009.

[10] A. Salado and R. Nilchiani, "Using Maslow's hierarchy of needs to define elegance in system architecture," Procedia Computer Science, vol. 16, pp. 927-936, 2013.

[11] A. M. Madni, "Elegant systems design: creative fusion of simplicity and power," Systems Engineering, vol. 15, no. 3, pp. 347-354, 2012.

[12] A. Salado and C. Salado, "Systems engineering practices exhibited in the creation of a film original score," INCOSE International Symposium, vol. 25, no. 1, pp. 1147-1158, 2015.

[13] J. Maeda, The Laws of Simplicity, Mit Pr, Cambridge, Mass, 2006.

[14] D. A. Norman, Living With Complexity, Mit Pr, Cambridge, Mass, 2010.

[15] P. Hekkert, D. Snelders, and P. C. W. Wieringen, "Most advanced, yet acceptable': typicality and novelty as joint predictors of aesthetic preference in industrial design," British Journal of Psychology, vol. 94, no. 1, pp. 111-124, 2003.

[16] R. Arnheim, Art and Visual Perception: A Psychology of the Creative Eye. The New Version, Revised Edition, University of California Press, Berkeley, 1974.

[17] M. Gell-Mann and S. Lloyd, "Effective complexity," in Nonextensive Entropy- Inderdisciplinary Apllication, pp. 387-398, Oxford University Press, Oxford, UK, 2004.

[18] W. R. Ashby, An Introduction to Cybernetics, Chapman \& Hall, London, 1973.

[19] A. Salado, L. Iandoli, and G. Zollo, "Painting systems: from art to systems architecting," INCOSE International Symposium, vol. 26, no. 1, pp. 773-787, 2016.

[20] T. McDermott and A. Salado, "Improving the systems thinking skills of the systems architect via aesthetic interpretation of art," INCOSE International Symposium, vol. 27, no. 1, pp. 1340-1354, 2017.

[21] B. Belke, H. Leder, and C. C. Carbon, "When challenging art gets liked: evidences for a dual preference formation process for fluent and non-fluent portraits," PLoS One, vol. 10, no. 8, article e0131796, 2015.

[22] C.-C. Carbon and H. Leder, "The repeated evaluation technique (RET). A method to capture dynamic effects of innovativeness and attractiveness," Applied Cognitive Psychology, vol. 19, no. 5, pp. 587-601, 2005.

[23] T. J. McCabe, "A complexity measure," IEEE Transactions on Software Engineering, vol. SE-2, no. 4, pp. 308-320, 1976.

[24] K. Sinha, Structural Complexity and Its Implications for Design of Cyber-Physical Systems, Doctoral Dissertation, Massachusetts Institute of Technology, 2014.

[25] J. R. C. Piqueira and S. H. V. L. de Mattos, "Note on LMC complexity measure," Ecological Modelling, vol. 222, no. 19, pp. 3603-3604, 2011. 
[26] L. Ji, W. Bing-Hong, W. Wen-Xu, and Z. Tao, "Network entropy based on topology configuration and its computation to random networks," Chinese Physics Letters, vol. 25, no. 11, pp. 4177-4180, 2008.

[27] J. Blijlevens, C.-C. Carbon, R. Mugge, and J. P. L. Schoormans, "Aesthetic appraisal of product designs: independent effects of typicality and arousal: effects of typicality and arousal," British Journal of Psychology, vol. 103, no. 1, pp. 44-57, 2012.

[28] D. E. Berlyne, "Novelty, complexity, and hedonic value," Perception \& Psychophysics, vol. 8, no. 5, pp. 279-286, 1970.

[29] R. Barthes e and S. Sontag, A Barthes Reader, Hill \& Wang Pub, New York, 1985.

[30] H. Kawabata and S. Zeki, "Neural correlates of beauty," Journal of Neurophysiology, vol. 91, no. 4, pp. 1699-1705, 2004.

[31] D. M. Buede and W. D. Miller, The Engineering Design of Systems: Models and Methods, John Wiley \& Sons, Inc., Hoboken, NJ, 2016.

[32] R. J. Cloutier and D. Verma, "Applying the concept of patterns to systems architecture," Systems Engineering, vol. 10, no. 2, pp. 138-154, 2007.

[33] D. L. e. Z. Scott, A Primer for Model-Based Systems Engineering, Vitech Corporation, USA, 2011. 


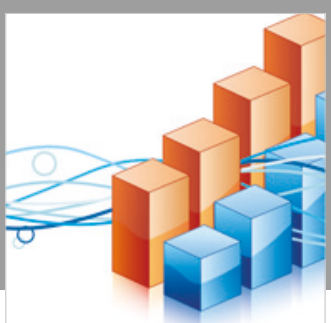

Advances in

Operations Research

\section{-n-m}
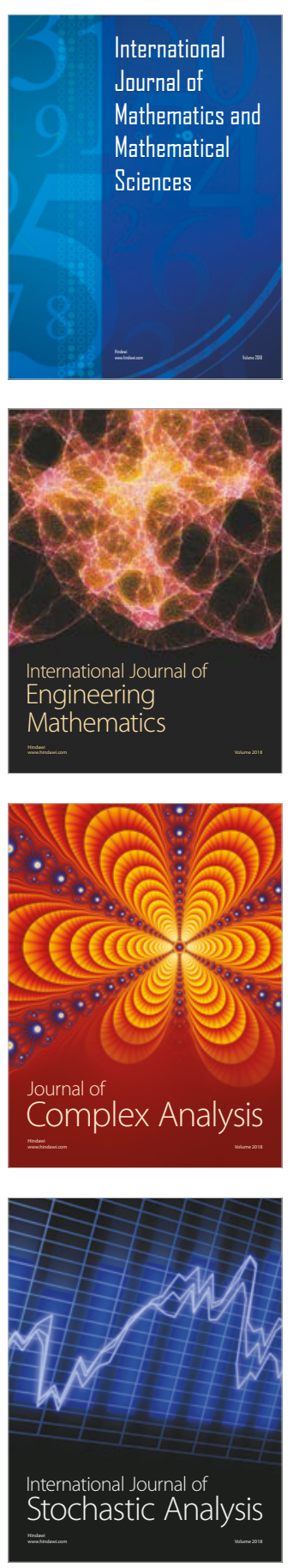
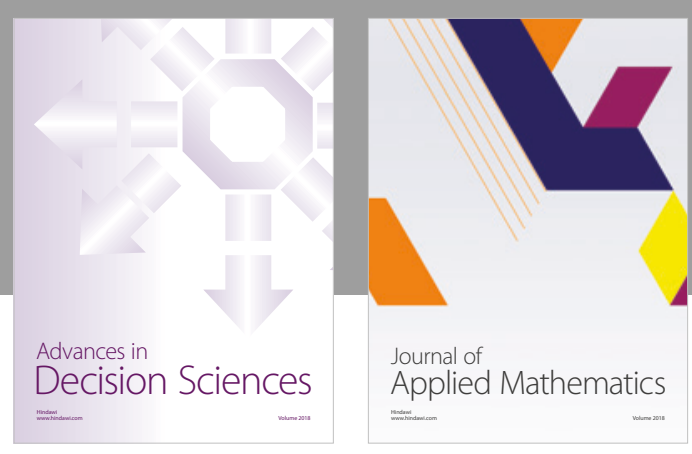

Journal of

Applied Mathematics
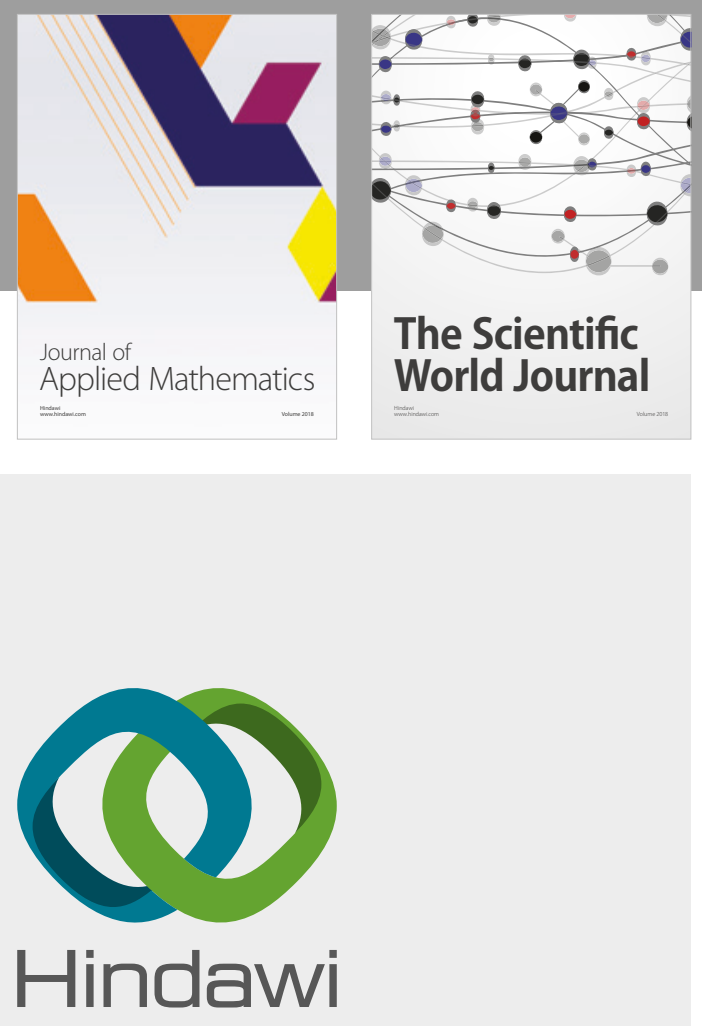

Submit your manuscripts at

www.hindawi.com

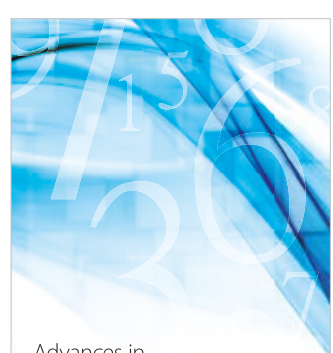

Advances in
Numerical Analysis
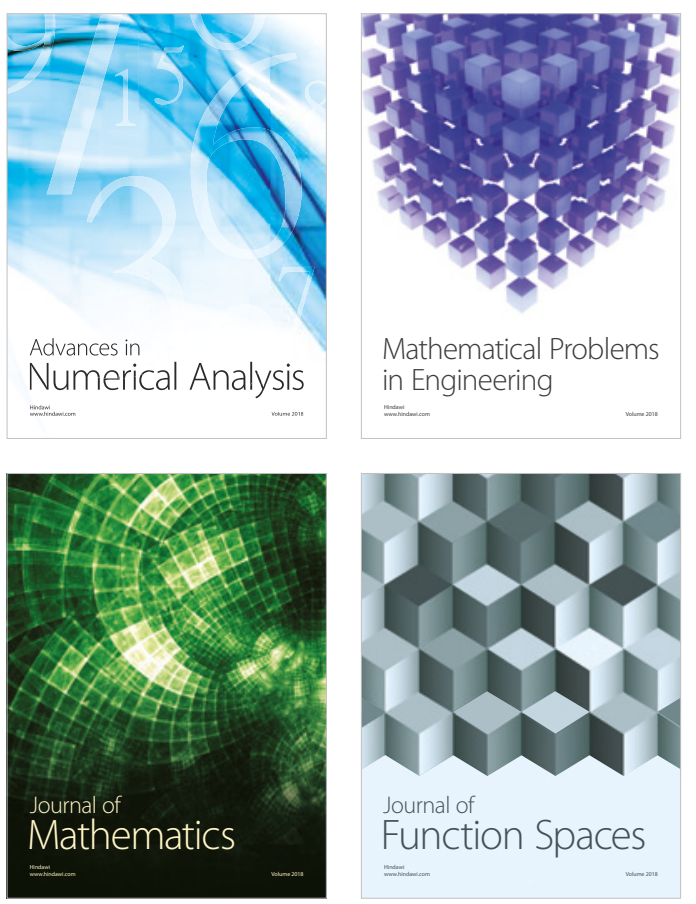

Mathematical Problems in Engineering

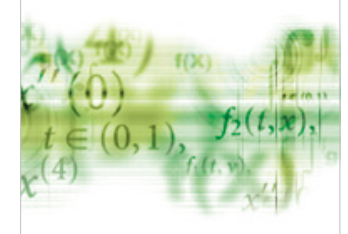

International Journal of

Differential Equations

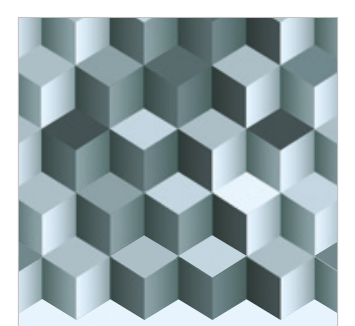

Journal of

Function Spaces
The Scientific

World Journal

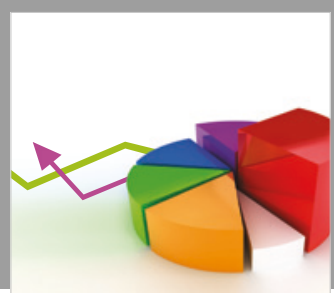

Journal of

Probability and Statistics
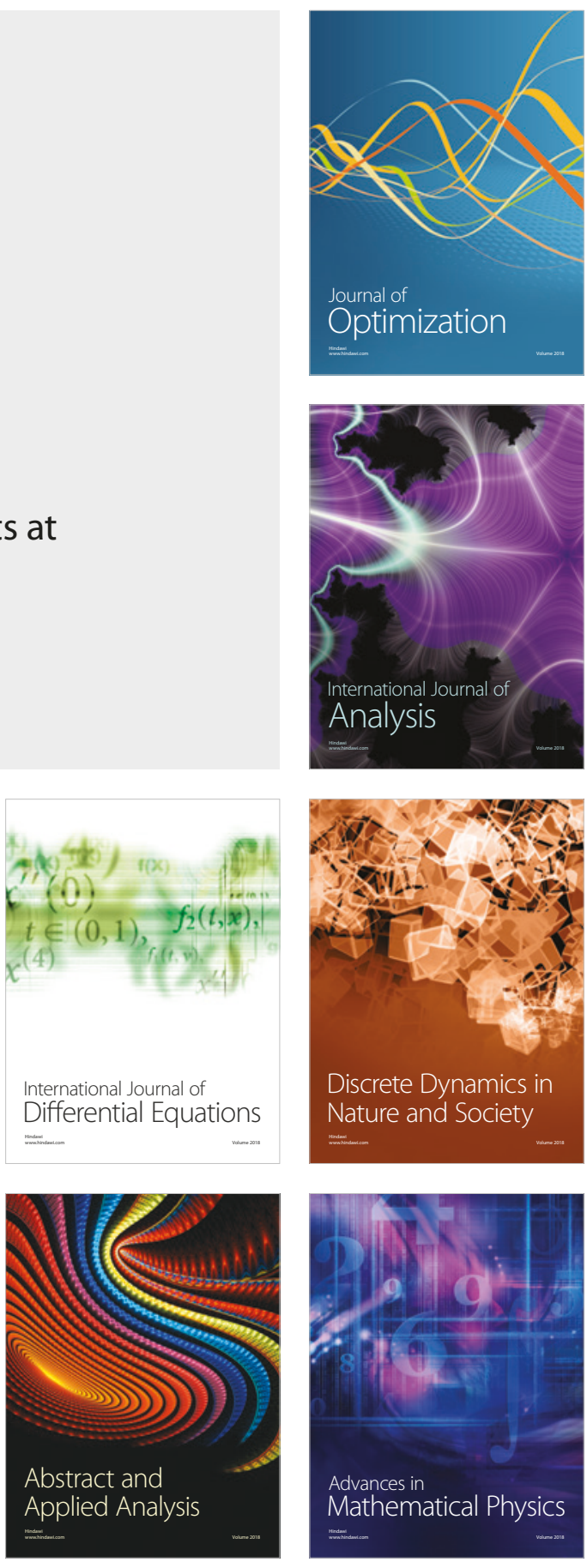